\title{
Propuesta metodológica para el análisis de una realidad agraria*
}

\author{
Cooperación Técnica Francesa*
}

\section{Introducción}

Las sociedades rurales representan, a menudo, para los gobiernos y sus tecnócratas, un mundo "misterioso", "tradicional", o "atrasado", difícilmente controlable, cuyas respuestas a las políticas agrícolas parecen imprevisibles.

La realidad no corresponde, 0 no obedece, a los suefios tecnocráticos: la historia reciente nos enseña que la mayoría de los proyectos de desarrollo agrícola en el Tercer Mundo, cualesquieras que sean sus bases teóricas o ideológicas, resultaron ser un fracaso.

"...Se atribuye con frecuencia el fracaso de gran número de estos proyectos al desconocimiento que tienen sus autores de las realidades agrarias..." (7): desconocimiento de la complejidad de los sistemas de producción, de sus origenes y de sus relaciones con la formación social global, $y$, por consiguiente, ignorancia de sus racionalidades socioeconómicas.

Parece que El Salvador no escapa a esta experiencia general y que los diagnósticos existentes previos a cualquier intervención (proyecto, política agrícola, Reforma Agraria,....) no han logrado generalmente entender las prácticas campesinas, su evolución pasada y su racionalidad socioeconómica presente: las estrategias nacionales de desarrollo agricola, además de favorecer en primer lugar el dinamismo del latifundio

- Este estudio constituye la primera parte de un trabajo más completo sobre el Análisis de un Sistema Agrario a partir del caso del Distrito de riego de Zapotitán.

** Este trabajo fue elaborado por los miembros de la Cooperación Técnica Francesa.

- Clement San Sebastián,

- Jean Marc e Isabelle Touzard,

- Stephane Canu,

- Benoit Faivre. 
agroexportador, han seguido, después de la segunda guerra mundial, dos líneas principales: "Revolución verde"* y extensión agrícola. Si se ha notado como consecuencia un aumento sensible de los rendimientos para los granos básicos, esencialmente el maíz entre los años 1965 . 1972, apareció rápidamente un agotamiento de estas políticas agrícolas, por haber querido difundir técnicas supuestamente "superiores y modernas" sin tomar en cuenta la estructura social y agroecológica en que esta inmerso el agricultor.

Frente a esta exigencia de analizar una realidad agraria en su globabilidad, antes de cualquier intervención y dado el vacío metodológico correspondiente, la Misión Francesa de Apoyo a la Universidad Centroamericana José Simeón Cañas escogió llevar a cabo una propuesta de un método de análisis de una realidad agraria, y aplicarlo al caso de Zapotitán, treinta kilómetros al Oeste de San Salvador. **

Este proyecto de riego que ya parecía conocer muchas dificultades para mantenerse, constituyó nuestro campo de investigación y de ensefanza, logrando la integración de los estudiantes a este trabajo sin disponer de muchos recursos logísticos y financieros. Además, al incluir este estudio en un proyecto de investigación más amplio del departamento de economía, queriamos traer un aporte nuevo en cuanto al análisis de proyectos agrícolas, y proponer algunas reflexiones alrededor de la problemática agraria, "epicentro" de la crisis salvadoreña actual.

\section{Objetivos y justificación del estudio}

\section{La Problemática económica y agrícola salvadoreña}

\subsection{La crisis del modelo de acumulación salvadorefía}

La guerra civil que vive El Salvador es en parte una consecuencia de la imposibilidad con la cual se enfrenta el modelo socio económico nacional en seguir desarrollándose sin generar graves desequilibrios. Ya se mostró ("Dinámica y crisis de la economía salvadorena", Departamento de Economía de la UCA, ECA enero febrero 1986) que la estructura económica que se ha construido en el país, basada en la agroexportación y después completada por un aparato industrial dependiente del exterior, no ha podido dar un crecimiento sostenido y equilibrado (y todavía menos podrá hacerlo visto la coyuntura internacional actual).

En el origen de la acumulación de capital encontramos al monocultivo

- Caracterizada esencialmente por la introducción de nuevas variedades con alta potencialidad genética.

* Los resultados de esta investigación forman el contenido de la segunda parte de este artículo, a parecer en próximo número de Realidad Económica y Social. 
del café bajo una forma de latifundio, estando estrechamente articulado con el minifundio productor de granos básicos.

La rapidez del proceso de acumulación originaria en El Salvador se puede atribuir en gran medida al alto grado de complementariedad existente entre los dos sistemas de producción: Durante la estación lluviosa (invierno), el minifundista se dedica al cultivo de granos básicos para conseguir la mayor parte de su alimentación y la de su familia, mientras que en el verano, al no poder cultivar maíz o frijol, por la falta de agua, se emplea para el corte del café, consiguiendo así un ingreso monetario complementario necesario para cubrir sus necesidades.

Esta organización presenta para el latifundio dos ventajas básicas:

1. El latifundio puede mantener una tasa de ganancia elevada gracias a un costo en mano de obra mantenido bajo, o sea que la fuerza de trabajo se reproduce afuera de su unidad productiva a un costo inferior, al costo de una mano de obra asalariada a tiempo completo.

2. La complementaridad temporal entre los dos sistemas de producción permite la reproducción y una cierta estabilidad interna del sistema latifundista.

Despues de la segunda guerra mundial, el crecimiento nacional se apoyó también sobre otras actividades: El cultivo del algodón y una industrialización de "Sustitución de importaciones":

-El cultivo del algodón, por su parte, retomó las mismas características que la caficultura, al nivel de sus relaciones con el minifundio, empleando la mano de obra minifundista para la cosecha en verano (la mecanización de la cosecha es además prohibida por la ley).

-Por otra parte, la industrialización naciente generó rápidamente graves desequilibrios principalmente por:

- La ausencia de producción de bienes de equipo y de materias primas industriales, que solamente modificó la estructura de las importaciones.

- La ausencia de un mercado interno solvente y homogéneo, que la podría estimular durablemente: Si se observan las múltiples experiencias de industrialización en la historia, se puede constatar que el crecimiento de los jóvenes aparatos industriales se hizo casi siempre en base a un mercado interno, o sea que contaban con una demanda efectiva capaz de proporcionarles una dinámica básica para su desarrollo. En el caso salvadoreño, el latifundio agroexportador poderoso y sus sectores dependientes (beneficios, instituciones financieras) han tendido siempre a limitar la canasta básica de la mayoría de la población a un mínimo incompatible con las necesidades de mercados internos para el aparato industrial. 
La búsqueda de estos mercados en la integración centroamericana, logró para un corto tiempo, sustituir a la demanda interna, rechazando momentáneamente la resolución de esta contradicción fundamental; pero el fracaso del proyecto económico centroamericano, y el papel creciente del gasto público en la demanda efectiva nacional (que trataba también de complementar a la demanda privada) han rapidamente amplificado los déficits fiscales que no se podian acumular sin problemas.

Este modelo de acumulación "excluyente" y extrovertido, que queda subordinado a la producción agroexportadora y que por eso, en el caso salvadoreño, supone la existencia de una mano de obra numerosa y barata, no ha podido responder al crecimiento demográfico rural que por no encontrar un espacio por lo menos de sobrevivencia, produjo una crisis agraria profunda y desestabilizadora para el sistema económico y social salvadoreño.

\subsection{La imposible reproducción del Agro.}

Se puede estimar que en 1986, las familias campesinas sin tierra, y las que tienen menos de 5 hectáreas representaban alrededor de 1,6 millones de personas, poseyendo solamente el 10 por ciento del área agrícola útil del país (4). Este conjunto social importante, que produce más del $85 \%$ de los granos básicos, está marginado por el régimen de acumulación vigente en el país. No puede constituir un polo de acumulación dadas las limitaciones con las cuales tiene que enfrentarse, entre ellas:

1. Los precios de venta de sus principales productos, además de experimentar una fluctuación anual que el no puede manejar por la debilidad de sus medios de almacenamiento, no han incrementado en relación con el aumento de los precios de los insumos. Para el maíz, por ejemplo, hemos calculado que entre 1975 y 1986, los costos de producción fueron multiplicados por 3.2, mientras que el precio de venta lo fue solamente 1.6. Las políticas agrícolas no favorecen a los pequenos productores que deben afrontar la competencia de una ayuda alimentaria enorme y ahora estructural (que genera, además mecanismos de sustitución) y que tienen que contentarse con un salario agrícola real que ha sido dividido por 3 desde el 1979 (14).

2. El acceso a la tierra es muy limitado, tanto a nivel de cantidad como de cualidad, puesto que el latifundio está ubicado en las mejores tierras.

3. También este acceso es muy inseguro; los arrendatarios de pequeñas parcelas no tienen ninguna seguridad acerca de las posibilidades de seguir cultivando la misma tierra año tras año, al no ser protegidos por una legislación precisa. Esta inestabilidad obviamente tiene con- 
secuencias en el deterioro de los suelos, puesto que el agricultor, en estas condiciones, no tiene ningún interés en tomar medidas para conservar la fertilidad de los suelos.

4. Tampoco la organización del sistema bancario nacional da al pequefio campesino la oportunidad de tener acceso al crédito, por las excesivas garantías pedidas. El no puede entonces realizar las inversiones que le permitirian incrementar su producción. Esto impone al minifundista la necesidad de utilizar los servicios de los intermediarios usureros, los "coyotes", que además de otorgar un crédito de avio se encargan de la comercialización de las cosechas y a menudo del abastecimiento en insumos, obteniendo ganancias en cada operación.

Esta ausencia de control del minifundista sobre los principales determinantes de su producción, y la falta de una política claramente en su favor, explica por qué el no puede esperar romper con una lógica de simple sobrevivencia, que se traduce al nivel macroeconómico por el bloqueo de la demanda interna.

Además, es preciso reubicar este análisis en un esquema explicativo dinámico: La reducción física del acceso a la tierra para los pequefios productores, debido al proceso de apropiación por el latifundio y al crecimiento demográfico, ha generado rápidamente una crisis de reproducción de las condiciones agronómicas de sus cultivos: La fertilidad no se puede reconstituir a causa de la desaparición del retorno forestal, del barbecho, y de la imposibilidad de realizar una transferencia de fertilidad con el ganado (no se puede tener pastos, y el ganado representa una inversión importante poco accesible). Eso se acompaña a menudo con una desestructuración del suelo, de una erosión creciente y de la proliferación de plagas (por la sucesión de los mismos cultivos). Los rendimientos tienden a bajar; los hijos crecen sin posibilidad de seguir cultivando su tierra; el entorno socio-económico le desventaja cada día más; de ahi, el se enfrenta con las siguientes alternativas:

1. No hay posibilidad de encontrar una tierra virgen por la ausencia de frontera agrícola en el país y además Honduras no lo es más desde la "guerra del futbol".

2. Puede seguir cultivando una tierra alquilada más fértil, dejando su pedazo al barbecho. Sin embargo, esta alternativa es mucho más difícil desde la implementación del decreto 207, por el miedo que tienen los propietarios, y representa un costo alto.

3. Puede mantener, o aumentar, sus rendimientos con el uso masivo de abono químico y de productos fitosanitarios.

Esta alternativa, muy adoptada en los años 60 , con la tentativa de "revolución verde", necesita un cierto nivel de liquidez a menudo 
inaccesible, y es más y más difícil, dado el aumento de los costos de producción.

4. La incomoración en una cooperativa constituye una posibilidad limitada: Los socios son en primer lugar los antiguos asalariados 0 colonos de la finca expropiada, y la organización social de la producción no corresponde siempre a sus intereses.

Además no puede esperar necesariamente mejorar sus condiciones de vida, vistas las limitaciones con las cuales se enfrentan las cooperativas de la fase I: deuda agraria, alta proporción de tierra de baja calidad, etc.

5. Se puede volver en asalariado casi permanente, cayendo en una estrategia inmediatista, cultivando el pedazo que le deja el propietario, o las orillas de las carreteras.

6. La búsqueda de una actividad no agrícola en San Salvador (en el sector informal) viene a ser a menudo la solución más interesante. Los trabajos que puede efectuar cada miembro de la familia, proporcionan generalmente un ingreso total superior al sueldo de un trabajador agrícola. La crisis de reproducción de la economía minifundista, amplificada por los efectos de la guerra que ella alimenta, es el motor del crecimiento de la capital, verdadero cáncer para la economía salvadorena. El "rechazo" del mundo rural (a causa de la crisis) se articula con la "llamada" de la economía urbana, sostenida por el sector público y la ayuda extema.

7. Por fin, la emigración hacia los Estados Unidos representa quizás la mejor alternativa para el pequeño productor, o sus hijos: podrá así sostener el sistema de producción de su familia mandando dólares regularmente.

Esta crisis de la economía campesina, resultado de la no adecuación del modelo de acumulación nacional con las potencialidades agroecológicas y las necesidades de desarrollo económico y social del país, tiene graves consecuencias que amenazan seriamente el futuro de EI Salvador:

-El medio ecológico se deteriora año tras año: la deforestación y la sobreexplotación de suelos frágiles se traducen por una erosión importante y una desestabilización climática (el árbol es un regulador hídrico, el albedo cambia, etc...).

- La producción alimentaria se agota, y logra cubrir solamente una parte del consumo real (que es inferior al consumo requerido para una dieta de $2200 \mathrm{Kcal}$ por día). En 1985, 70\% en el caso de los cereales, 
$56 \%$ para la leche,... en forma de ayuda internacional (PL 480, PMA,...)

- La miseria nural es profunda: El porcentaje de desnutrición para los niños menores de 5 anos que ingresaron en las consultas externas del ministerio de salud en 1982 era de $64 \%$ en las zonas rurales (y $59 \%$ en las zonas urbanas) (16). Ya hemos visto que esta marginalización se materializa con el bloqueo del desarrollo económico y por conflictos sociales que vive el país.

-El subempleo general afecta al país en el agro y en las ciudades, mientras existen espacios subexplotados en las fincas privadas o cooperativas.

Estas tendencias parecen bastante peligrosas porque corresponden a fenómenos que se extienden sin control real y que son muy difíciles de contrarrestar.

La reforma agraria que se implementó a partir de 1980 no cambió profundamente la problemática del agro, al ser parcial y tímida en sus lineamientos. Al contrario, por la existencia de una deuda agraria elevada, tanto las cooperativas de la fase I como los beneficiarios del decreto 207 se encuentran a menudo en una situación morosa que compromete su sobrevivencia financiera. Además las cooperativas han conservado los mismos criterios de gestión que las antiguas fincas, considerando la ganancia al final del ejercicio como objetivo principal contable.

Frente a este primer planteamiento general se ve la necesidad de buscar y proponer alternativas económicas para el país. Si las posibles parecen articularse alrededor de dos proyectos globales -el primero correspondiendo a una economía subordinada completamente a la influencia norteamericana, el segundo a una economía más autocentrada e integrada al espacio centroamericano-, la problemática agraria quedará siempre en un lugar estratégico para el país.

Eso implica la urgencia de iniciar una reflexión seria y lo más amplia posible, acerca de esta crisis agraria y de las políticas agrícolas que la podrían regular 0 afrontar.

\section{El proyecto del Departamento de Economía y el papel de la agricultura en un modelo de desarrollo alternativo}

Cuatro siglos de "desarrollo" basado sobre la extroversión del país y la concentración del capital en pocas manos, han mostrado cómo este modelo crea profundas desigualdades y desequilibrios macro-económicos, minimizados actualmente por la ayuda externa, pero revelados

- Cálculos personales 
por la guerra. Frente a esta crisis y sus causas, el Departamento de Economía de la UCA ha redactado un proyecto global de investigación, intitulado "Hacia la Estabilización a través del Desarrollo Endógeno", que quiere proponer alternativas económicas viables para el país, privilegiando la reactivación de la economía interna para resolver el problema del desempleo masivo actual y asegurar una mayor equidad distributiva. La problemática consiste en conocer si "...es posible reactivar la economía en el corto plazo impulsando los sectores productores que atienden demandas de los sectores de menores ingresos, sin rebasar los límites que los desequilibrios macro-económicos imponen a la reactivación económica", teniendo en perspectiva las articulaciones con el mediano y largo plazo.

Una de las hipótesis del proyecto del Departamento es que ningún desarrollo económico del país puede ser posible sin una reactivación prioritaria del sector agrícola, que podría conducir, en el corto plazo a la creación de empleo, valor agregado y demanda efectiva popular, y constituir en el mediano plazo, una fuente de financiamiento endógena, para los otros sectores económicos.

Las metas de esta rama agroalimentaria del proyecto son:

- Hacer un diagnóstico general del modelo agroalimentario actual de El Salvador, para poder sacar conclusiones en cuanto a lo que ese sector debe y puede cumplir dentro de las alternativas de reactivación económica propuestas por el equipo de investigación.

- Analizar la diversidad de los sistemas de producción del sector agrícola, reubicándolos en el entorno económico nacional e internacional; identificar los que cumplirían con la reactivación económica definida más arriba, y definir sus limitaciones.

Un principio fundamental de este trabajo es que ninguna política agraria puede ser viable sin un análisis preliminar profundo de la realidad agraria, de las lógicas de funcionamiento y de los factores limitantes de los sistemas de producción que se quiere sostener o desarrollar.

\section{3. "El Proyecto de Zapotitán" dentro de este programa de Investigación.}

En ese marco, el diagnóstico del sistema agrario de Zapotitán y la evaluación de un proyecto de riego y avenamiento de larga amplitud, tiene un papel importante. Es un primer intento de:

-Proponer una metodología de acercamiento a una realidad agraria que pueda realmente dar un conocimiento global del funcionamiento y de los factores limitantes de la zona, y mostrar la dinámica y las racionalidades de los distintos tipos de sistemas de producción iden- 
tificados. Este estudio pues, quiere brindar un método de investigación cuyos resultados deben permitir llevar a cabo intervenciones que respondan a las necesidades reales de los agricultores. La evaluación exante de un proyecto de riego que esta lejos de cumplir con sus objetivos iniciales, puede mostrar que una de las condiciones necesarias al éxito de cualquier política agrícola alternativa es el conocimento en profundidad de los sistemas de producción presentes.

- Estudiar en sí misma una alternativa para el pais, el riego. En efecto, frente al crecimiento de la población, y la limitación del área agrícola útil del país (limitación geográfica por la erosión y por el conflicto), hay una necesidad de intensificar la producción por manzana. En el cuadro de una política agrícola que quiere romper con los desequilibrios y la dependencia alimentaria, el riego constituye una alternativa de incremento en el empleo y en el valor agregado nacional por activo (empleado o no empleado), siempre y cuando se organicen las condiciones o los cambios estructurales necesarios en el agro y en su entorno económico social.

Dentro de estas condiciones que permiten una buena adopción de una alternativa tecnológica como el riego, por las poblaciones locales, y de acuerdo con los objetivos macroeconómicos, podemos distinguir:

- Características de los sistemas de producción (organización del trabajo, tamaño de las unidades de producción etc.).

- Condiciones económicas de la zona considerada: modificacón del acceso a los medios de producción, de la organización del mercado y del crédito, protección del medio natural etc.

- Condiciones intrínsecas a la tecnología: para que no aumente la dependencia de los sistemas de producción, ni la del país, y que sea adecuada a las especificidades del medio.

- Condiciones sociales para que una dinámica social efectiva y amplia sostenga la innovación; toma en cuenta de las organizaciones presentes, libertad de expresión...

Entre otras zonas de riego, hemos escogido como campo de estudio el Distrito de Riego y Avenamiento № 1, Zapotitán, por varias causas materiales:

-Es el único proyecto de riego y drenaje estatal de larga amplitud que fue realizado y que consiguió algunos resultados, aunque está muy lejos de cumplir con sus metas iniciales. fácil.

-Está cerca de San Salvador, en una zona no conflictiva, y de acceso

- La zona fue el cuadro de numerosos informes; los primeros datan de los años 60, cuando fue emitida por el gobierno la licitación para el 
proyecto de riego y drenaje, y las últimas son un perfil agroeconómico del Distrito de Zapotitán, realizado en el 1982 por el IICA y el MAG. Todos esos informes constituyen ya una base de datos, de cualidad muy heterogénea, pero que nos han permitido ganar bastante tiempo.

Además el Ministerio de Agricultura y Ganadería tiene en sus "gavetas" varios proyectos de riego que hasta ahora no han sido realizados por falta de recursos, pero que podrian aprovecharse de la experiencia de Zapotitán.

\section{Propuesta metodológica para el análisis de la realidad agraria}

\section{La necesidad de retornar al "objeto estudlado"}

Mientras que la agricultura constituye el sector productivo más importante de El Salvador y el "epicentro" de su crísis actual, la investigación en economía agricola en este pais adolece de un retraso teórico profundo, y se encuentra recargada por un cierto dogmatismo. Este campo está polarizado alrededor de dos núcleos antagonistas: Un enfoque marxista que extrae su esencia teórica de la "cuestión agraria" "de Kautsky" y un enfoque neoclásico que se esconde detrás de una ensenanza tecnicista y funcionalista.

El análisis Kautskiano, concebido en Europa frente al estallido del campesinado durante la revolución industrial, ha encontrado un campo muy propicio en América Latina para explicar la penetración del capitalismo en el agro. Este enfoque afirma que la competencia entre estructuras desiguales (distinta composición orgánica del capital) implica una concentración del capital en la agricultura y una proletarización inevitable de la mayoría campesina. Además de sacar conclusiones todavía no verificadas sobre la base de reflexiones incompletas de Marx ( $s e$ afirma la superioridad de las grandes explotaciones, sin tomar en cuenta el nivel de desarrollo de las fuerzas productivas...), a menudo, los campesinos son considerados como una clase reaccionaria y atrasada, más o menos homogénea, y que debe desaparecer rápidamente. Tales análisis reduccionistas y sometidos a estereotipos intelectuales, subrayan por lo menos la necesidad de relacionar el acercamiento económico con el análisis histórico concreto.

El enfoque neoclásico, ampliamente desarrollado por universidades norteamericanas y adoptado en América Latina por la mayoría de los tecnócratas y por los sectores ligados a la empresa privada, radica en un modelo explicativo que hace abstracción de la historia, de las estructuras, al mismo tiempo que ignora los comportamientos desiguales y las relaciones sociales: El juego de la oferta y de la demanda para los pro- 
ductos agrícolas, en relación con las ganancias de productividad, "debe" conducir a un aumento de la oferta, lo que implica una regulación por la baja de los precios y la eliminación hacia otros sectores económicos, de los productores no competitivos. Este análisis, a menudo no explicitado por los que lo utilizan, carece particularmente de aplicabilidad al estudio de las agriculturas del tercer mundo, supuestamente atrasadas. Además considera el progreso técnico como un elemento "natural" que se impone sin discutir sus vínculos sociales y apoyándose sobre un comportamiento empresarial.

Si bien existe en El Salvador algunas adaptaciones empiricas de estos dos núcleos teóricos rígidos, no se encuentra una construcción teórica basada en conceptos capaces de identificar la complejidad y la singularidad del agro salvadorefio. ¿En vez de perderse en debates universitarios que buscan adecuar lo "desconocido" al "conocido importado", no sería mejor concentrar sus esfuerzos alrededor de la elaboración de un método de análisis que permita entender en su globalidad el funcionamiento de una región agrícola? No se trata de negar la necesidad de las referencias teóricas sino de condenar el dogmatismo y abogar por un retorno al "objeto estudiado".

Antes de exponer nuestra propuesta metodológica, parece necesario examinar los diagnósticos "clásicos", determinar algunas de sus limitaciones prácticas que explican, a menudo, el fracaso de los proyectos que los han seguido.

\section{Reflexlones criticas acerca de los métodos de análisis clá- sicos.}

Frente a la necesidad de establecer un diagnóstico, especialmente para los proyectos financiados por instituciones internacionales, se elaboró poco a poco métodos para analizar el medio rural, y para llevar a cabo evaluaciones ex-ante. A menudo, estos métodos ocultan sus referencias teóricas neo-clásicas implícitas. Se trata desde hace poco de esconderlas detrás de una adaptación empírica.

\subsection{La primacia de la tasa de rentabilidad económica in- terna.}

La mayoría de las publicaciones que proponen estos métodos (las del $B I R D$, de la OCDE, por ejemplo) son destinadas más que todo a financieros, preocupados por la rentabilidad de sus inversiones. El medio rural se considera como una "caja negra" demasiado compleja para entenderla. Se intenta hacer la comparación entre "input" y "output" potencial, exprimiendo todos los criterios de análisis escogidos en valor monetario. Por eso, los autores parecen preocuparse más de la tasa de 
rentabilidad económica interna (tasa de actualización que anula la ganancia colectiva total neta actualizada), que de la comprensión efectiva de la realidad y asi de las consecuencias del proyecto afuera de la "caja negra". Esta obsesión por la tasa de rentabilidad interna lleva hasta organizar todas las modalidades del proyecto, con el objetivo de alcanzar una tasa definida antes del diagnóstico.

\subsection{Un modelo de explotación preconcebido.}

Estos métodos "clásicos" se basan generalmente sobre un modelo preconcebido y analizan la situación "antes del proyecto" únicamente con arreglo a este modelo. El proyecto va a construirse con referencia al modelo como si hubiera una homogeneidad, mientras que la realidad es diferenciada: En el caso de Zapotitán, un grupo de consultores había fijado, antes del análisis de la zona, un modelo de pequefro productor de hortaliza y ganadería de leche con un pedazo de una hectárea, sin tomar en cuenta la variabilidad del medio, la variabilidad de los productores, etc.; o sea era un modelo concebido teóricamente sin hacer referencia a la realidad. Además este modelo se refiere generalmente a un empresario potencial que buscaría maximizar su tasa de ganancia, cualquiera que fuera la organización de la producción, individual, estatal o cooperativa. Sin embargo esta racionalidad empresarial representa solamente una de las racionalidades que se encuentran en la realidad, donde los productores, según las condiciones socio-económicas y ecológicas existentes, tratan de minimizar los riesgos, maximizar la remuneración horaria del trabajo familiar y del margen bruto por hectárea, etc.

\subsection{Un diagnóstico parcializado y tecnicista}

Por eso, estos métodos de análisis consisten más que todo en una cosecha de las informaciones necesarias a la construcción del modelo. Se divide la realidad en varios estratos (medio físico, medio económico, medio humano,...), implicando conclusiones falsas o por lo menos incompletas, porque:

- Cada información está separada de su contexto.

- Cada información no está entendida en relación con otros fenómenos (sobre todo históricos).

El diagnóstico es, entonces, generalmente el producto de grupos de investigadores especializados y "compartimentados". Cada uno, por su lado, estudia su campo de especialización y eso llega a un catálogo de datos, que no es realmente explotable: El "Perfil del Distrito Zapotitán"10 representa el ejemplo característico de este tipo de diagnóstico, produciendo seis volúmenes de datos sin sacar una síntesis real. (Además comporta errores básicos y falsas informaciones que ocultan la pro- 
blemática real). Esta concepción que consiste más que todo en adicionar en vez de sintetizar, revela una visión tecnicista del desarrollo donde el hombre es solamente un sujeto, y la sociedad un cuadro no materializado por la existencia de relaciones sociales. Esta visión tecnicista está legitimada por la especialización científica y asimila generalmente el "proyecto de desarrollo" a un modelo de crecimiento identificado al modelo capitalista. En el "modo de producción capitalista," la tecnología alcanza un tipo de "fetichismo" que conduce a comprender esta técnica fuera de las relaciones sociales existentes y a atribuir a ella una función privilegiada, autónoma, apartada de la sociedad. Así mismo, en muchos países que buscan una transformación socialista, una visión tecnicista se ha mantenido como consecuencia de la no-toma en cuenta de las relaciones de producción existentes y como consecuencia de la competencia con el capitalismo.

No obstante, cualquier técnica, en todas las épocas históricas, es indisociable del sistema social en el cual se inscribe. Un diagnóstico que no toma en cuenta la articulación entre las fuerzas productivas (que incluye la tecnología, los medios de producción y la fuerza de trabajo) y las relaciones sociales de producción, es reductor y sirve más que todo a la ideología y al orden establecido. La dinámica social real habiendo sido ocultada, el proyecto que sigue es generalmente apropiado por grupos reducidos o a veces, rechazado por la problación.

\subsection{Un prejuicio tecnológico.}

Entonces, estos diagnósticos "clásicos" son a menudo orientados, finalizados, con el propósito de introducir un paquete tecnológico. Esta tecnología corresponde generalmente a la tecnología elaborada y adoptada en Europa y Norteamérica.

Su introducción no es siempre eficiente al nivel puramente técnico, dada la especificidad del medio tropical: En el caso de Zapotitán sólo cinco pozos de bombeo están funcionando en 1987, dentro de los 21 inicialmente construidos, por muchas razones de este tipo.

La introducción de esta tecnología es un costo inicial alto para el Estado e implica rápidamente un círculo vicioso de dependencia (por ejemplo, un tractor importado implica la importación de diesel, repuestos, máquinas adaptadas, etc.). Este costo no es siempre compensado por un aumento al nivel del valor agregado por la población en edad de trabajar. Por ejemplo, en un país como El Salvador donde no hay posibilidades de levantar la producción agrícola con una extensificación y donde, ya existe una alta tasa de desempleo o subempleo productivo, la introducción de un tractor, permite aumentar la productividad del trabajo del beneficiado, pero, reemplazando el trabajo de quizás veinte per- 
sonas, llega a un costo social alto, sin aumentar el valor agregado ni la producción total.

Además se busca promover esta tecnología sin que sean realizadas, por lo menos algunas condiciones socio-económicas que en Europa permitieron su éxito:

1) Una tecnología producida por una artesanía y después una industria nacionales, que podía ser introducida progresivamente según las capacidades de absorción de las unidades de producción.

2) Un incremento relativo del costo de la mano de obra, debido a la llamada de parte de la industria, necesitando un aumento de la productividad del trabajo agrícola.

Un diagnóstico que, por 10 menos, no estudia el referencial técnico local, las prácticas de los agricultores y las condiciones socio-económicas que pueden bloquear la difusión de una tecnología naciente, no concuerda con un desarrollo económico nacional real. En el caso de Zapotitán, los diagnósticos no han estudiado el sistema de riego campesino que existía "antes del proyecto" (1200 hectáreas por lo menos) y propusieron un sistema de riego más "moderno" que funciona actualmente a $20 \%$ de lo que era previsto.

\subsection{El cuantitativo más que el cualitativo}

Por otra parte los diagnósticos "clásicos" (handbook del Banco Mundial, por ejemplo) están generalmente obsesionados por el "cuantitativo", el "promedio" y la "representatividad". Se apoyan sobre encuestas exhaustivas o efectuadas en una muestra "representativa", aleatoria, sin estratificación. Después, ellas se tratan generalmente con análisis multidimensionales (A.C.P, ...) o regresivos lineales que buscan correlaciones entre algunos factores.

Este método implica una muestra importante, y entonces un costo alto, para llegar muchas veces a evidencias: No importa tanto saber que la tasa de correlación entre, por ejemplo, el área cultivada y la proporción de maíz cultivado pueda ser $-0.88, \delta-0.92$. Además este método puede ocultar casos típicos que tienen un interés al nivel de la investigación (innovadores, finca importante,...), que no tienen una representatividad numérica alta. No se trata de rechazar estas técnicas de encuestas y de proceso de los resultados, que pueden completar eventualmente un primer diagnóstico, pero los resultados obtenidos en función del costo y de la rápidez de su realización no dan una rentabilidad interesante.

En las últimas publicaciones institucionales que proponen métodos de análisis (3), se toma más en cuenta la necesidad de desarrollar estudios de casos cualitativos y profundizados, pero no aparecen bien 
articulados con el conjunto de la metodología propuesta. Por lo menos este cambio muestra la evolución profunda que sigue un organismo de financiamiento potente, como la BIRD, en el marco de la evaluación de proyectos. La necesidad de definir nuevos métodos de análisis para el medio rural motivó, desde hace diez años, investigadores y centros de investigación, como el grupo AMIRA (1) o el movimiento "investigacióndesarrollo" (que empieza a distinguirse en la investigación en economía agrícola (9)). La metodología que vamos a exponer es el resultado de estos debates recientes en relación con algunas experiencias propias (17), la última siendo la investigación desarrollada en el distrito de Zapotitán.

\section{Objetivos generales del diagnóstico.}

El diagnóstico que se establece antes de cualquier intervención (proyecto, política agraria,...) tiende siempre a orientarse en función de la naturaleza de la intervención. Parece necesario, por lo menos en un primer momento, alejarse de la finalidad institucional impuesta para buscar una comprensión global del funcionamiento de la zona previamente definida. Sin embargo, un diagnóstico no puede constituir una finalidad propia; tiene que integrarse en una perspectiva general de transformación de las condiciones de producción, cuyas metas prácticas tienen que ser definidas con los distintos grupos interesados (y por lo menos los agricultores!). En este sentido podemos fijar una serie de objetivos, articulados entre ellos mismos, que van a plantear los ejes del cuadro metodológico del diagnóstico.

\subsection{Un conocimiento global}

El objetivo central es el conocimiento global y sintético de la realidad agraria, conjunto ecológico utilizado y transformado por el hombre para asegurar su existencia. Pero cuando se dice global no se entiende "promedio" sino más que todo "conocimiento de la diversidad de esta globalidad": diversidad del medio, diversidad de las situaciones en donde se encuentran los productores. En este marco se trata de seguir un doble camino:

-El primero es más práctico: consiste en contestar a la pregunta: ¿Cómo nos representamos la sociedad rural y los sistemas de producción, tomando en cuenta esta diversidad?

-El segundo es más teórico: consiste en responder a una segunda pregunta: ¿Qué esquema explicativo y que funcionamiento se puede atribuir a estos sistemas?

Se trata entonces de conocer todo, sabiendo que es imposible: Lo 
esencial consiste en tener una visión general de las principales interacciones existentes.

\subsection{Buscar los factores limitativos}

El segundo objetivo, más preciso, consiste en identificar los factores que limitan la reproducción de las unidades de producción, pero siempre relacionando estos factores con la variabilidad, la diversidad de la realidad agraria: cada unidad de producción se encuentra con un conjunto de coacciones o factores limitativos, distintos según sú ubicación geográfica (rentas diferenciales,...) y sus características socio-económicas. Por eso tendremos que lograr estratificar el espacio (será la zonificación) y la población de productores (será la tipología) para llegar a conjuntos, o nubes, que tengan una cierta homogeneidad al nivel de estos factores limitativos.

\subsection{Mostrar la dínámica social}

Tendremos como otro objetivo de analizar las relaciones existentes entre los agricultores y revelar la dinámica social. Eso implica un estudio de la estructuración real de la sociedad rural con sus ejes de polarización (que no son siempre ejes de acumulación), sus distintos grupos u organizaciones (segmentos de linaje, comunidad, comité, sindicato, cooperativa,...) y los proyectos de sociedad que tienen. Se busca entender la organización social, que corresponde a las fuerzas productivas de la zona, subrayando los elementos motores de la dinámica social, los movimientos sociales que puedan ser los ejes de transformación.

\subsection{Revelar las contradicciones existentes.}

No es suficiente determinar los factores limitativos e identificar la organización social y su dinámica; conviene mostrar las relaciones que existen entre todos estos factores o fenómenos, o sea sacar a luz las contradicciones subyacentes que explican realmente los bloqueos socio-económicos. Se puede revelar por ejemplo:

-Contradicciones internas a las relaciones de producción

- Contradicciones entre la organización social y la extensión de una técnica o de un sistema de producción.

- Contradicciones entre acumulación al nivel de la zona y acumulación nacional o extranjera.

- Contradicciones entre producción alimentaria y agro-exportadora, etc. 


\subsection{Evaluar las tendencias históricas.}

Es una necesidad reubicar el acercamiento global de la zona agraria en una perspectiva histórica. Si entendemos efectivamente el estudio histórico como el elemento explicativo principal de la realidad agraria presente, tiene que ser también una contribución a la elaboración de los futuros posibles: ¿Cuáles son las tendencias a corto, mediano, largo plazo en cuanto a elementos determinantes para la reproducción del sistema agrario:

-Al nivel de la reproducción del ecosistema, anotando especialmente los problemas de erosión, conservación de la fertilidad, aumento de la densidad de población...

-Al nivel de la evolución de las interacciones entre las prácticas, técnicas y las relaciones sociales.

-etc...

\subsection{Hacer un primer Inventario de las alternativas 0 solu- ciones posibles.}

Finalmente el diagnóstico ya tiene que sacar algunas propuestas o alternativas posibles que permitirian superar los factores limitativos, resolver en parte las contradicciones expuestas, y anticipar o eventualmente enfrentar, una tendencia histó́rica. Por eso, se busca en primer lugar hacer el inventario de las soluciones posibles con los interesados, tomando en cuenta las distintas propuestas, y las soluciones que ya pueden existir (estudiando los elementos que pueden limitar su extensión).

Se busca también las alternativas que podrían ampliar el referencial técnico en el cual las comunidades rurales van a sacar soluciones para resolver sus limitaciones.

\section{Hipótesis y princlplos metodológicos del diagnóstico.}

Para alcanzar los objetivos definidos es preciso adoptar ciertas hipótesis y seguir algunos principios de base; eso no compone una receta que sería suficiente aplicar, sino que puede constituir una guía metodológica para un diagnóstico agrario, dejando un margen de adaptación en función de la especificidad de la realidad.

\subsection{Las hipótesis metodológicas tienen que ser bien cla- ras.}

Para llevar a cabo el método de análisis que proponemos, fijaremos algunas hipótesis metodológicas que deben siempre quedar bien 
entendidas, porque condicionan y limitan la representación de la realidad que queremos construir.

\subsubsection{Un enfoque sistémico.}

La necesidad de tener una representación sintética de la realidad llega a buscar un instrumento metodológico que permita reunir los elementos más diversos de una realidad agraria, sean estos físicos, biológicos o sociales, en una misma teoría analítica. El concepto de sistema trata de responder a esta exigencia, organizando el conocimiento en esquemas de funcionamiento globales: "Un sistema es un conjunto de elementos que tienen interrelaciones entre sí y que actúan hacia un mismo objetivo. El estudio del Sistema abarca no solamente el estudio de los elementos en sí, sino también, y sobre todo el estudio de las interrelaciones entre estos elementos" (18)*. Esta visión globalizante, que permite enfrentar la parcelación científica, va a identificar a distintas escalas, entidades funcionales donde se puede estudiar, por ejemplo, las interacciones entre elementos técnicos y elementos sociales.

-En una primera escala, podremos identificar, como base estructural de una realidad agraria, las UnIdades de Producción que pueden ser definidas como entidades que tienen un cierto margen de decisión y de manejo en cuanto a la producción agrícola, pero que pueden, ser diferentes de unidades de consumo o de acumulación, etc... Estas unidades (ya sean unidades campesinas, empresariales, cooperativas, estatales...) son caracterizadas por su Sistema de Producción, definido como "la combinación más o menos coherente en el espacio y en el tiempo de ciertas cantidades de fuerza de trabajo y de distintos medios de producción (tierra, máquina, riego, insumos,...) con miras a obtener distintas producciones"7.

Este concepto de sistema de producción, que puede ser dividido en sub-sistema de cultivos (al nivel de cada parcela) y sub-sistema de ganadería (al nivel de cada hato) va a explicar el proceso de producciónreproducción de la explotación subrayando sus itinerarios técnicos y agricolas, tomando en cuenta sus relaciones con el exterior (coacciones de factores socio-económicos influyentes).

De hecho, el uso del concepto de sistema de producción supone que se podrá encontrar una cierta racionalidad que se explicará con la hipótesis de coherencia entre las prácticas del productor confrontando a una serie de factores limitativos y la representación que el se hace de la realidad.

- Consideramos el término "objetivo" como la apropiación de parte del productor de una determinación socio-económica "objetiva" y no en su sentido ideológico de elección "libre". 
-Al nivel de la Región o de la Zona Agrícola vamos a identificar el Sistema Agrario que será la representación construida de la realidad.

Esto se puede definir teóricamente como el "conjunto de las técnicas, de los comportamientos y relaciones económicas y sociales establecidos en una sociedad, y su espacio, en cuanto al uso de la tierra para la producción agrícola" (15). La definición de este espacio puede parécer relativamente imprecisa, visto que sus criterios de delimitación no son constantes (pueden ser el relieve, el modo de ocupación humana y agrícola, etc. ...). Sin embargo, este espacio corresponde a una cierta unidad geográfica reconocida desde mucho tiempo por los geógrafos, y va a tener más que todo un papel operacional para el diagnóstico. El uso del concepto de sistema agrario subraya la importancia de las relaciones entre los distintos sistemas de producción. Así, por ejemplo se puede

DIMENSION EXPLICATIVA

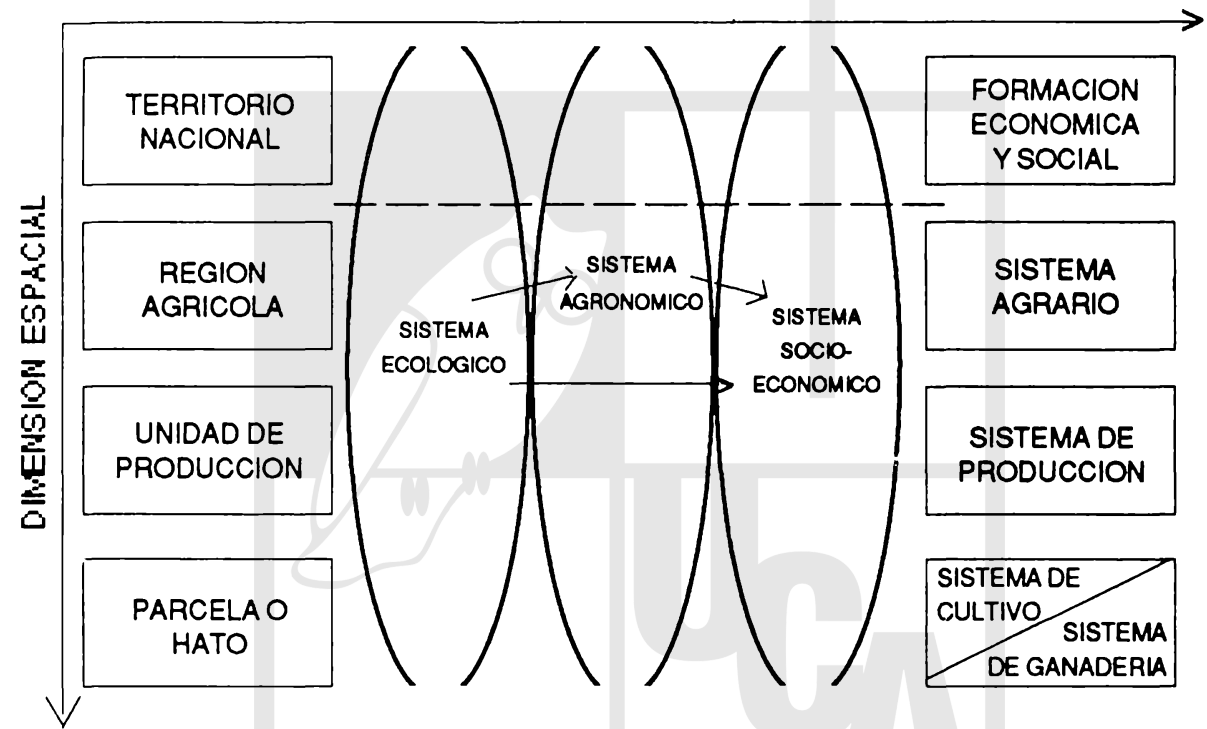

ESQUEMA N 1: LOS CONCEPTOS SISTEMICOS UTILIZADOS

representar el binomino "Latifundio-Minifundio" con todas sus interrelaciones, sus "complementaridades" (a los niveles geográficos, del uso de la fuerza de trabajo del destino de la producción...), y con su polarización socio-económica extrema, en favor del latifundio.

El análisis del sistema agrario se hace, entonces, estudiando la dinámica de los distintos sistemas de producción en relación con el medio agro-ecológico, con la organización social existente y con la in- 
fluencia determinante del entorno externo del sistema (o sea la formación económica y social nacional y sus interdependencias internacionales). En la noción misma de sistema agrario se consideran, entonces, los distintos niveles de la organización de la actividad agrícola que corresponden a distintas escalas espaciales, como sistemas encajados e interrelacionados entre ellos.

En otra dimensión, más explicativa, se puede dividir el sistema agrario en 3 sub-sistemas:

-El sub-sistema ecológico, constituido por las características ambientales típicas de la zona, determina "potencialidades y limitaciones naturales".

-El sub-sistema agronómico reune los mecanismos adoptados por el hombre para transformar el medio, con el fin de una producción agrícola.

- El sub-sistema socio-económico revela las condiciones socioeconómicas que permiten un beneficio del medio.

Estos subsistemas cruzan los conceptos más globalizantes de sistema de producción y de sistema agrario. Las interrelaciones entre estos distintos conjuntos constituyen un punto importante de análisis, y sobre todo del enfoque histórico (ver esquema 1).

\subsection{Hipotesis de coherencia de los productores}

La segunda hipótesis metodológica general que hacemos, plantea que los seres humanos que actuan en nuestro sistema agrario, tienen una coherencia en su comportamiento, o sea que tienen "buenas razones para hacer lo que hacen". Las prácticas en el agro no son cosa de brutos o de atrasados, como creen algunos técnicos sino que existen razones objetivas $y / 0$ subjetivas que explican estos comportamientos. Esta coherencia tiene que ser entendida en relación con los objetivos que siguen los productores (ingreso monetario, seguridad, tiempo de trabajo, actividad social,...); y estos objetivos van a depender esencialmente de las condiciones ecológicas, económicas y sociales que rodean el productor: En última instancia, la existencia de un sistema de producción es determinada por las condiciones económicas objetivas que le permiten existir.

Si un campesino, por ejemplo, cultiva de tal manera, con tal objetivo principal, es porque se encuentra con una cierta disponibilidad de recursos (tierra, fuerza de trabajo), ciertos riesgos climáticos, cierto acceso al mercado y a la información,... De otra manera, se puede decir que el productor aguanta un conjunto de factores limitativos precisos, que determina fuertemente su sistema de producción. Por consecuencia, se puede hacer la hipótesis de racionalidad de un sistema de producción, 


\section{Un ejemplo de racionalidad socio-económica de un slstema de producclón: \\ el caso del pequeno campesino haitiano}

...El pequeno campesino de las montanas haitianas, acostumbrado desde hace ya mucho tiempo a los intercambios comerciales, pero sometido a las múltiples presiones de los comerciantes y los usureros, quiere sustraerse a dichos intercambios y se esfuerza por lo tanto, él también, por producir en sus tierras la máxima cantidad de víveres para su autosubsistencia, recurriendo lo menos posible a los servicios de los comerciantes. Las asociaciones de cultivos de plantas comestibles son la regla general. Cereales, tubérculos y leguminosas alimentarias cubre la casi totalidad de las superficies cultivadas. El cafeto, que era antano el principal cultivo de renta, está en regresión constante. Las fluctuaciones de los precios entre el momento en que el campesino, cuyos medios de almacenamiento son muy reducidos, vende su cosecha, y en el que debe volver a comprar viveres para su subsistencia, son generalmente muy amplias: de 1 a 3 el mismo ano para los frijoles. El agricultor trata pues de llevar a cabo sistemas de producción que le permitan cosechar productos durante todo el ano. Los cultivos asociados, cuyos ciclos pueden ser de duración muy diferente unos de otros, responden por una parte a esta preocupación. Pero sobretodo, los campesinos de la zona montanosa tratan de cultivar en los diferentes pisos ecológicos, aunque tengan que tomar tierras en aparcería, para diversificar los calendarios de cultivos y disponer de cosechas en todo momento. Esta práctica requiere trabajo durante todo el año en la explotación, sin interrupción; se manifiesta también por desplazamientos a pie, a menudo considerables; pero la mano de obra familiar es abundante, las posibilidades de empleo en el exterior son muy reducidas (poco industrias, nada de artesanía), los agricultores consideran que su propia fuerza de trabajo tiene un costo de oportunidad nulo. La concentración parcelaria de las explotaciones, propuesta por ciertos expertos intemacionales, se revelaría, en esas condiciones, particularmente destructora. No olvidemos que los sistemas de producción escalonados de esa forma permiten mal que bien la supervivencia de una población muy densa, casi siempre superior a 250 habitantes por kilómetro cuadrado. El problema esencial reside sin duda, hoy en dia, en el carácter precario de la tenencia de la tierra. La necesidad urgente de tierras cultivables en las diferentes altitudes incita a los campesinos a tomar parcelas en arriendo o en aparceria, 
por períodos cortos, mediante el pago de una renta muy importante (equivalente, muy a menudo, a la mitad de la cosecha). Los costos elevadísimos de los trabajos de agrimensura y de las escrituras notariales disuaden a las familias de proceder al reparto de las tierras en el momento de las herencias. Numerosas parcelas permanecen indivisas y las disputas son muchas para su utilización.

El carácter precario de la tenencia prácticamente no incita a los campesinos a acondicionar y mantener el patrimonio territorial sobre las tierras de las que no son directamente propietarios; el sobrepastoreo es muy frecuente, los acondicionamientos anti-erosivos son dejados abandonados, y las restituciones orgánicas son inexistentes. Los resíduos de cultivos se trasladan sistemáticamente hacia las parcelas en propiedad. Las transferencias de fertilidad se hacen aquí, por lo tanto, de las parcelas cuya tenencia es precaria hacia aquellas de las que los campesinos disponen con toda seguridad por un período largo (huertos caseros)...

en MARC DUFUMIER: Sistema de producción y desarrollo agrícola en el tercer mundo. C.I.P.C.A. Penú.

tomando en cuenta sus recursos propios, su entorno socio-económico sabiendo que la reproductibilidad de sus condiciones de existencia son determinadas por las leyes económicas objetivas.

Esta hipótesis de racionalidad nos incita a buscar el "porque" de una práctica, de una técnica; pero, como cada hipótesis, tiene que ser confirmada o invalidada.

La realidad muestra, a veces, un cierto nivel de lo que definimos como irracionalidad económica: es cierto que cada productor, en su cuerpo social, se encuentra integrado en un proceso de reproducción de las experiencias y prácticas agronómicas de sus precedecesores, lo que implica la existencia de un cierto tiempo de adaptación pragmática cuando surge un cambio importante en su entomo. Además, esta coherencia del productor agrícola no tiene generalmente el referencial explicativo "científico" que utiliza el agrónomo, pero sus bases pragmáticas experimentales, explican la lógica interna de su sistema de producción. De todas maneras, una irracionalidad económica importante se traduciria directamente con su "eliminación" como productor agrícola, sería sancionada históricamente.

\subsubsection{Hipótesls sobre una clerta homogeneldad de com- portamiento}

La tercera hipótesis general que hacemos, supone una cierta 
homogeneidad de los comportamientos humanos cuando el grupo social es homogéneo. Eso significa que los productores que se encuentran en condiciones parecidas de un punto de vista ecológico, económico y social, tienden a actuar de la misma manera, con los mismos objetivos, con una misma racionalidad. De una manera más precisa podemos decir que los productores tienen por lo menos las mismas potencialidades a partir de limitaciones comunes. Eso resulta de la hipótesis según la cual los factores objetivos determinan los comportamientos en última instancia.

Esta última hipótesis va a justificar la construcción de una tipologia que representará las distintas situaciones típicas y sus racionalidades correspondientes.

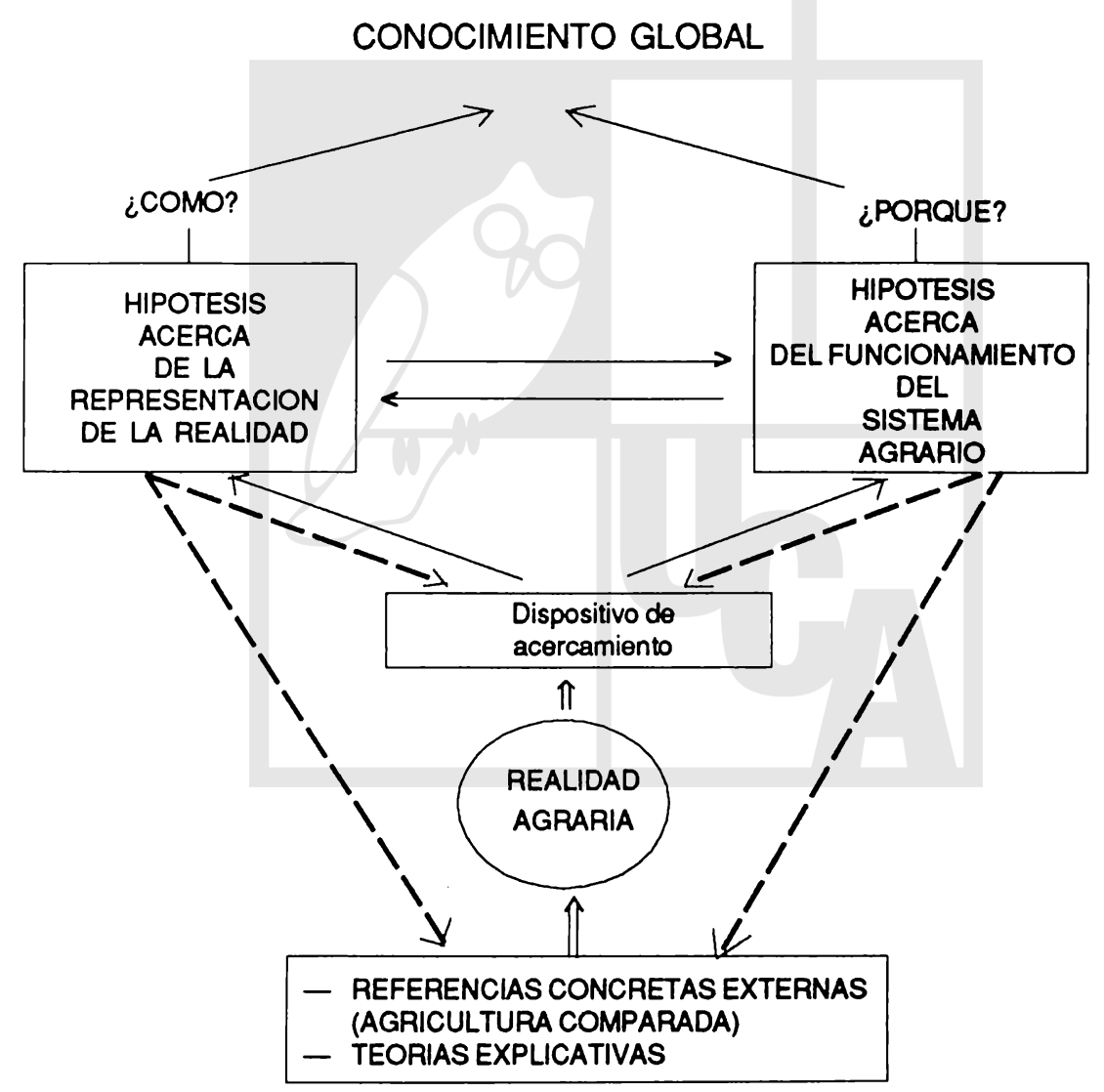




\subsubsection{Un acercamiento pluridisciplinario y dinámico.}

El análisis sistémico, instrumento metodológico que trata de responder a la necesidad de un conocimiento global y sintético de la realidad agraria, debe traducirse al nivel del grupo de investigación.

La complejidad del "objeto estudiado", el sistema agrario, implica recurrir a disciplinas tan diversas como la sociología, la economia, la agronomía, la phyto-ecología,etc... A pesar de que estos distintos campos científicos tienen conceptos propios, es preciso imponer intercambios constantes en el seno del equipo para enfrentar la parcelación criticada anteriormente.

Las preocupaciones del equipo de trabajo deben orientarse alrededor de un análisis histórico para entender, por ejemplo, la lógica de evolución entre las prácticas técnicas y las relaciones sociales.

\subsubsection{La variabilidad en primer lugar.}

En cada etapa del diagnóstico nos impondremos de estudiar, siempre y en primer lugar, las diferencias pertinentes. Si el promedio tiene su importancia al nivel macroeconómico, es conveniente partir de la diversidad concreta para entender los factores, explicativos de cada realidad. Este principio nos incita a descubrir, en el espacio como en el cuerpo social, situaciones típicas contrastadas caracterizadas por distintas lógicas de funcionamiento. No se trata de negar lo cuantitativo, sino de decir que tiene sentido solo si esta relacionada con lo cualitativo.

\subsubsection{Un acercamiento iterativo.}

El conocimiento de la realidad agraria sera progresivo y de acuerdo a hipótesis que afinaremos poco a poco. Las primeras hipótesis en cuanto a la representación del sistema agrario serán confrontadas con la realidad, lo que las modificará de nuevo. Este proceso constante de verificación esta naturalmente orientado, y eventualmente transformado, por las hipótesis que el engendra. Este tipo de "ida y vuelta" se establece también entre la representación hipotética de la realidad (¿Cómo?) y la explicación de su funcionamiento (¿Porqué?) que utiliza referencias teóricas y comparaciones con otras realidades. Este procedimiento iterativo (ver el esquema $\mathrm{N}^{2} 2$ ) constituye la primera base de cualquier acercamiento científico de un medio y puede ser seguido también para la negación de hipótesis que eran adoptadas hasta ahí.

\subsubsection{Un método participativo.}

Llevar a cabo un diagnóstico participativo no es solamente una exi- 
gencia ideológica o moral, es una exigencia científica: tenemos que integrar los actores del sistema agrario en la elaboración del diagnóstico, porque son ellos que lo conocen lo mejor posible, quizás de manera no organizada, incompleta y con referencias explicativas subjetivas. Es preciso incorporar los productores en el proceso iterativo de verificación de las hipótesis formuladas ¿Qué piensan ellos de estas suposiciones, explicadas en un lenguaje accesible?

El "diálogo problematizador"8 es el mejor medio para revelar los mecanismos de determinación en el sistema agrario y entender las racionalidades de los productores. Naturalmente tenemos que relativizar las respuestas en función de la posición social del encuestado y del sesgo que llevamos:

- Se trata de darse cuenta del sistema de referencias explicativo, o de la "ideología", del productor y de relacionarlo con su posición social.

- No se debe olvidar que el encuestado nos pueda tomar por controladores o al contrario por portadores de una ayuda externa potencial.

De todas maneras el diálogo que se establece, o la coparticipación, debe valorizar el productor encuestado, el cual puede ayudar a la progresión del diagnóstico, si se le restituye los resultados parciales de la investigación.

\section{Las etapas del dlagnóstico.}

Después de haber fijado los objetivos que asignamos al diagnóstico, y los principios de base adecuados, es conveniente proponer de manera más concreta, el método de acercamiento que hemos tratado de aplicar en nuestra investigación. Hemos distinguido etapas que tienen una cierta independencia metodológica, pero que se articulan entre ellas y que, en la práctica, se desarrollan a menudo en el mismo tiempo.

\subsection{Un primer acercamiento general.}

La primera etapa del diagnóstico del sistema agrario, ya delimitado geográficamente, es una fase de acercamiento general que puede durar poco tiempo, pero que es fundamental. Esta "inmersión" en el medio consiste más que todo en cosechar y clasificar informaciones ya existentes, completándolas con algunas observaciones en el campo.

Al final de esta etapa se podrá identificar la diversidad de los sistemas de producción y recoger sus principales factores limitantes. Además, esta fase metodológica debe permitir caracterizar las unidades de observación (¿Cómo se define en la zona el concepto de unidad de producción?)

Del éxito de esta fase va a depender el desarrollo de las otras etapas y la duración del diagnóstico. Se trata de llegar lo más pronto posible a la 
constitución de una primera base de datos acerca del sistema agrario y a la elaboración de las primeras hipótesis que van a orientar las etapas siguientes. Por eso utilizaremos juntamente tres instrumentos principales de recolección de datos y de observaciones.

-Bibliografía ya existente.

Se impone de recoger el máximo de datos bibliografícos, cartográficos, datos estadísticos, monografías y cualquier informe en relación con la zona. Esta bibliografía habiendo sido realizada dentro de un contexto dado, para un objetivo determinado, siempre se debe relativizar una información en función de su origen. Por ejemplo, el "Perfil IICA-MAG de Zapotitán"10, al no tomar en cuenta los productores de cana del distrito, oculta la problemática de la estructura agraria; esto es característico de un diagnóstico institucional que no quiere volver a discutir la estructura establecida. De manera general la bibliografía estará mucho más valorizada cuando se tengan referencias concretas del terreno.

- Entrevistas con interlocutores escogidos.

En primer lugar, es necesario platicar abiertamente con los actores que ya tienen un conocimiento general de la zona. En prioridad se trata de los ancianos productores, testigos de las trasformaciones del sistema agrario, pero también de algunos técnicos o funcionarios (del Ministerio de Agricultura, del Banco Agrícola,...) de curas y notables. Muy a menudo, estos contactos son "pasajes" obligatorios para ser aceptados como investigadores en la zona y constituyen muestra de la estructuración social.

\section{—Primero "Trayectos"}

Es necesario algunos primeros recorridos, que traten de atravezar la heterogeneidad espacial, familiaricen al investigador con el medio agroecológico, las técnicas, etc..., y le proporcionen referencias concretas para elaborar las primeras hipótesis de trabajo.

\subsection{Zonificación y caracterización general del sistema agra- rio.}

Esta segunda etapa tiene como objetivo la caracterización general de la región de estudio, revelando su variabilidad geográfica. Se busca identificar las subzonas principales y definir para cada una de ellas la problemática agraria que se plante: la definición de estas zonas se hará en función de criterios precisos que influyan en los sistemas de producción y sus racionalidades, y que determinan realmente la variabilidad geográfica, identificada rápidamente en la primera etapa. Estos criterios corresponden no solamente a variables socio-económicas. La selección 
de ellas es por lo tanto, particular a cada sistema agrario, pero se escogen usualmente dentro de los siguientes:

- Cristerios físicos: pluviometría, temperatura, vientos, altitud, pendiente, tipo de suelo,...

- Criterios agro-ecológicos: uso del suelo, cobertura boscosa, manejo técnico,...

- Criterios socio-económicos: densidad de población, infraestructura y servicios, tamaño de fincas...

Para cada criterio escogido, se elabora un mapa, a partir de mapas ya existentes y de observaciones personales: En el caso de Zapotitán, por ejemplo, se necesitó solamente tres días para establecer el mapa de uso del suelo.

Paralelamente cada criterio está analizado cualitativamente tratando de entender los mecanismos de determinación que genera. Por ejemplo, un criterio como la pendiente determina fuertemente el nivel de erosión, las posibilidades de arado y por lo tanto influye en las prácticas agrarias. Es decir, estos estudios temáticos de los distintos factores naturales y humanos tienen como meta mostrar la importancia de cada uno de ellos como factor explicativo potencial (o sea tratar de jerarquizar sus papeles al nivel de la determinación de los sistemas de producción). Este intento descansa en una primera negación epistemológica del monodeterminismo: Es importante identificar los margenes de determinismo y los margenes de indeterminismo; no obstante, la existencia misma de los sistemas de producción identificados debe incitarnos a buscar sus fundamentos económicos.

Uno de los problemas que se plantean es la elección de los límites; es preciso que correspondan a un cambio importante en el determinismo de los sistemas de producción.

Por ejemplo, si estudiamos siempre la pendiente, se puede escoger, de acuerdo a las observaciones, un primer límite que corresponda a una sensibilidad a la erosión (puede ser, según el tipo de suelo, alrededor de $5-8 \%$ ) y un segundo límite que corresponda a una erosión crecida pero más que todo a una imposibilidad física de labrar (puede ser alrededor de $30 \%)$.

La superposición gráfica de los distintos mapas permite a continuación delimitar numerosas subzonas sintéticas pero que quedan todavía hipotéticas. Se puede ya visualizar algunas correspondencias o correlaciones geográficas que eventualmente revelan una relación de causalidad (por ejemplo estructura de la propiedad de la tierra y orientación productiva, ...). Pero se necesita verificar la pertinencia de la zonificación establecida con un método comprobatorio, constituido por la realización de trayectos o recorridos pertinentes; estos son de dos tipos: 
- Trayectos que van a atravezar la variabilidad máxima definida, o sea que tratarán de identificar la realidad de las transiciones entre las subzonas. Puede ser, por ejemplo un recorrido que "corta" un valle (si la altitud y la pendiente son determinantes), una vía de comunicación, etc...

- Trayectos que, al contrario, van a verificar la homogeneidad de una zona definida y comprobar que no se olvidó un factor determinante.

En este proceso de verificación, el paisaje agrario es el "interlocutor": consideramos que el paisaje registra los factores naturales, culturales y económicos; es a la vez receptáculo e integrador. La identidad de una estructura aparente, o tipo de paisaje se presenta pues como un espacio homogéneo a una cierta escala de percepción. Es decir que a cada zona definida debe de corresponder un tipo de paisaje agrario y/o una problemática específica.

Para salir de la construcción un poco clasificadora, tenemos que jerarquizar los distintos factores y lograr así una zonificación razonada y representativa. Eso implica a menudo la eliminación o la agrupación de algunas zonas o criterios definidos anteriormente. En cada zona adoptadas definitivamente, se esboza una problemática dominante que permitirá "interpretar las acciones que realizan los agricultores y entender el razonamiento que los impulsa a tomar determinadas decisiones en el manejo de sus fincas" (5).

El intento de "compartimentar" el espacio puede encontrar su limitación en el margen de impresición de las fronteras entre las zonas. Quizás sería más preciso adoptar un método de análisis espacial que, para cada tema estudiado, identifica conjuntos espaciales distintos, que tienen intersecciones entre ellos, como lo propone Ives LACOSTE ${ }^{12}$. Cada conjunto sería determinado por un criterio más sintético (zona de influencia de una ciudad, zona de colonización rural, cadena montañosa), y la diversidad se entendería como consecuencia de las intersecciones entre los numerosos conjuntos. Este método, quizás más justo, pero más completo, está en realidad mucho más adecuado al análisis geopolítico que al estudio de un sistema agrario. Por lo menos, parece suficiente relativizar la zonificación definida, tratando de acercarse lo más posible de la diversidad del paisaje, sin vacilar en modificarla más tarde.

\subsection{La historia agraria.}

La caracterización rápida y la zonificación permiten revelar algunos elementos estructurales del sistema agrario pero no su dinámica. El estudio histórico se impone como acercamiento capaz de explicar la situación actual y de sacar las grandes tendencias. El diagnóstico de un proyecto puede ser una contribución a la comprensión de los futuros posibles. 
De hecho, la diversidad determinada con la zonificación no tiene su explicación unicamente en un supuesto "determinismo natural", pero sobretodo en un determinismo socio-histórico articulado con las condiciones ecológicas.

El enfoque utilizado quiere integrar de manera global los elementos dinámicos de los diversos sistemas de producción logrando una convergencia de las ciencias sociales y agro-ecológicas, a los niveles micro como macroeconómicos. Por eso es importante concentrar la historia agraria alrededor de dos ejes:

-Un estudio de la evolución de la formación económica y social, del contexto nacional e internacional, porque generalmente son eventos externos al sistema agrario que provocan sus transformaciones.

- Un estudio de la transformación de los sistemas de producción en relación con la evolución de la organización social; es fundamental descubrir las compatibilidades o incompatibilidades entre las fuerzas productivas (y más específicamente la tecnología), y las relaciones sociales.

Este proceso, que toma en cuenta la diferenciación geográfica y social, permite analizar estados sucesivos del sistema agrario. Conviene conocer como se hace el pasaje de un estado al otro, aunque no se pueda afirmar luego la existencia de etapas bien definidas. Por lo menos, las distintas etapas de la historia agraria conocen cierto nivel de estabilidad, o una "velocidad" más o menos constante (el "pasaje" pudiendo ser entendido como una aceleración de la historia). El estudio histórico se desarrolla en base a referencias bibliográficas y a encuestas abiertas con los ancianos, siguiendo una guía establecida. Esta fase se constituye poco a poco, paralelamente a las demás (zonificación y tipología).

\subsection{Tipología de los productores}

La tipología se entiende como el producto de la diferenciación histórica de los sistemas de producción. Por lo tanto, es una visión dinámica de los sistemas de producción actuales, que tienen su origen en la historia y que se encuentran constantemente en un proceso de transformación.

Un análisis preciso del "cuerpo social" del sistema agrario -y por lo menos sus productores- es una necesidad para un diagnóstico. Numerosos proyectos o políticas agrarias han fracasado por haber presupuesto la existencia de un productor "promedio" que no solamente no corresponde a la realidad sino que simplemente no existe.

$\mathrm{Si}$ se pretende entonces lograr un mejor conocimiento del funcionamiento de la zona estudiada, es necesario construir una tipología 
que identifique los diferentes objetivos económicos de los productores, es decir los diferentes tipos de racionalidad económica que coexisten en este sistema agrario.

Esta tipología, que se quiere dinámica, va a ser relacionada con los factores que determinan las racionalidades económicas de los sistemas de producción desarrollados (en base a la hipótesis expuestas en el capítulo III).

Estos factores que son estructurales o históricos se encuentran dentro de los siguientes:

- Disponibilidad en medios de producción: tierra fuerza de trabajo, herramienta, agua,...

- Tenencia, tipo de acceso a los medios de producción: tierra, herramienta en propiedad, alquilados, en mediería, acceso a la fuerza de trabajo, relaciones de propiedad y de distribución.

- Condiciones del mercadeo:

Mercadeo de insumos, de la producción, de los bienes de consumo, crédito...

- Riesgos existentes:

Ecológicos (sequia,...), socio-económicos (mercado, inflación, guerra...).

Combinando y jerarquizando estos criterios según el impacto aparente que tienen (eso estando definido en la etapa precedente), se puede construir un cuadro de análisis, que va a ser el eje principal explicativo de la tipología dinámica o por lo menos el campo estructurado y limitado de las potencialidades "objetivas" que orientan las racionali-

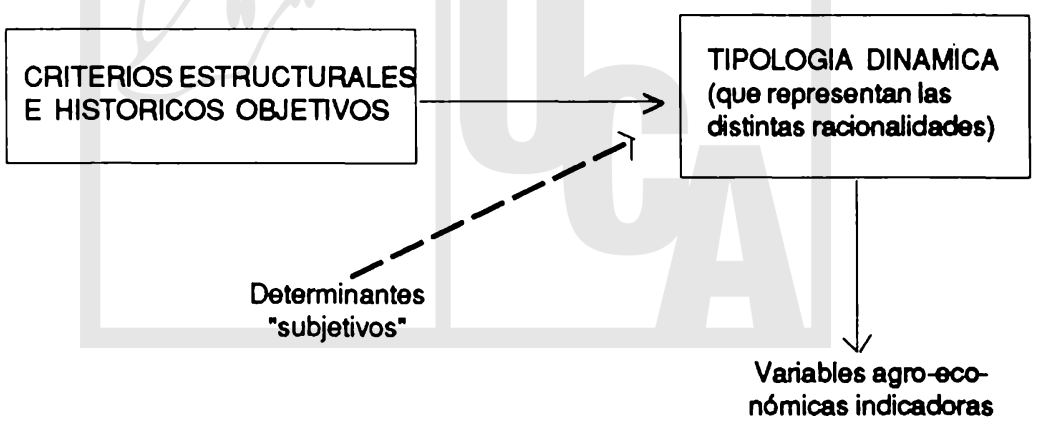

dades. Es cierto que la realidad de los comportamientos depende también de elementos de la historia personal del productor (instrucción,...) y de su integración dentro de la dinámica social (referente cultural, prestigio); pero los podemos considerar como determinantes más subjetivos y a menudo secundarios. 
Por otra parte, la tipología dinámica puede ser realizada directamente por algunas variables agroeconómicas que sintetizan el objetivo perseguido, esquematizado por un proceso de maximización (generalmente del factor más raro) o de minimización (de riesgos,...). Estas variables son por ejemplo: el margen bruto* (o neto** si las amortizaciones son importantes) por hectárea, la remuneración del trabajo familiar, la tasa de ganancia, la producción principal y su nivel técnico,... No obstante estas variables indicadoras no son explicativas, sino que son consecuencias de las racionalidades a identificar.

El esquema anterior puede resumir lo que acabamos de explicar:

Los criterios obejtivos, que permitiran llegar a los tipos y subtipos de la tiplogía dinámica, van a ser confrontados con la realidad agraria mediante las encuestas y modificada poco a poco en un proceso iterativo. Se alcanza de hecho a conjuntos de factores correlacionados que van a definir casos típicos, según la especificidad del sistema agrario. Efectivamente, los tipos definidos se entienden no como conjuntos compartidos del cuerpo social sino más bien como puntos de referencias alrededor de los cuales se distribuyen las unidades de producción, con combinaciones posibles.

La pertinencia de estudiar estos casos típicos, centros de gravedad de una nube de casos semejantes, reside en la voluntad continua de privilegiar el explicativo sobre el descriptivo, el cualitativo sobre el cuantitativo.

En este sentido se establece un muestreo estratificado, razonado y no aleatório, con arreglo a los criterios objetivos completados por algunos "criterios indicadores". Solamente algunas unidades de producción son escogidas en cada estrato definido, la cualidad de las encuestas y la elección pertinenente de los casos compensando el tamano reducido de las encuestas: estas encuestas muy completas abarcan varios aspectos como los datos estructurales, la historia de la explotación, los itinerarios tecnicos detallados con las interrelaciones entre actividades y resultados económicos, las relaciones de producción, etc..., (ver la guía de encuesta en anexo 1).

La desventaja de este método reside en que no nos permite sacar conclusiones estadísticas exactas en cuanto al número de fincas cerca del tipo. Sin embargo resulta ser un inconveniente muy relativo, frente a la cualidad de la información proporcionada. Además, se puede estimar la "representatividad" de cada tipo, combinando distintas estadísticas ya existentes (en función, por ejemplo de la correlación entre un tipo y algunos criterios objetivos).

- Producción agropecuaria total menos los costos de producción proporcionales.

** Margen bruto menos las amortizaciones anuales del activo fijo. 


\subsection{Dinámica social y otros estudios más especializados.}

La tipología, parte central del diagnóstico que ha diferenciado las distintas dinámicas y factores limitantes de los sistemas de producción y que permite prever ya las reacciones posibles a una intervención en el medio, ubica difícilmente estas explotaciones en un esquema relacional global. Por eso, conviene analizar también los distintos niveles de relación entre los "actores del sistema", identificados a través de la complejidad del cuerpo social. Este estudio es particularmente importante para definir eventualmente el nivel de intervención social de un proyecto.

Se trata generalmente de estudiar el funcionamiento de distintas unidades relacionales de la zona: Unidad de linaje o famiiar, unidad geográfica $o$ de vecindad, unidad asociativa o gremial,... Esta etapa se basa más que todo sobre un método de análisis en "racimo" que permite, a partir de un actor, subir a una unidad relacional.

La finalidad del diagnóstico puede implicar también el desarrollo de estudios más especializados, para completar el conocimiento general adquirido. Son, por ejemplo, estudios de mercadeo, estudios sobre los problemas de conservación de alimentación, etc... Pero queda siempre que estos diagnósticos temáticos sirven solamente si se integran en un diagnóstico global del sistema agrario.

\section{Conclusión}

El uso de este método permite caracterizar el medio natural y socioeconómico (su heterogeneidad, sus ventajas y apremios), reconocer los distintos tipos de sistemas de producción existentes, identificar sus cuellos de botella y sus relaciones con la economía global.

Además, este método de análisis de una realidad agraria es una herramienta de previsión, indispensable para anticipar los efectos de una intervención de toda clase (proyectos de desarrollo, políticas de intervención del estado), y para tomar decisiones.

Por eso, tipología e historia forman una unidad de análisis: cualquier tipología de sistemas de producción necesita la historia para ser explicativa, y los hechos históricos deben de ser clasificados, jerarquizados, y concentrados en una tipología.

En comparación con estudios de tipo estadísticos, este método tiene la doble ventaja de poder ser utilizado sin una abundancia de datos cuantitativos (muy a menudo escaos en el Tercer Mundo), y de necesitar una inversión en trabajo relativamente débil. 


\section{BIBLIOGRAFIA}

1. AMIRA: - Le suiviévaluation dans les projets de développement rural"- Paris, $2^{\mathrm{e}}$ odition, 1984

2. BETTELHEIM Charles: - Planification et croissance accélerée"- Maspéro, Paris, 1964.

3. BIRD: - Manuel sur le suivi et $1^{2}$ évaluation des projets de développement agricole et rural" -1982

4. CONDECORA, Comité Técnico, en base a información del I.C.N., 1986

5. Coopeación Técnica Francesa: - Estudio sistémico de la realidad agraria de una micro-región de Costa-Rica, Nicoya, Hojaancha"- Escuela de Ciencias Agrarias, UNA, San José, 1986.

6. DUFUMIER Marc: -"El Salvador; La Question Agraria" - in Problèmes d'Amérique Latine, Documentation Francaise № 4830, 1987.

7. DUFUMIER Marc: - "Sistemas de producción y desarrollo agrícola en el Tercer Mundo"- CIPCA, PIURA, Perú, nov. de 1985.

8. FREIRE Paolo: - ¿"Extensión o comunicación? La concientización en el medio rural"od. siglo XXI, México, 1972.

9. GRET: -"Démarches de la recherche-développement appliquées au secteur de la production rurale dans les pays en voie de développement". Paris, 1983.

10. IICA-MAG: -"Pérfil del Distrito de ZAPOTITAN" - VI volúmenes, San Salvador, 1982.

11. IFARC: - " cahiers de la formation professionnelle á la recherche en milieu rural des régions chaudes, fascicule № 3 : Méthode d'enquète en milieu rural", volumen 1 , Montpellier, 1982.

12. LACOSTE Ives: - "Unité et diversité du Tiers-Monde" - La Découverte, Paris, 1984.

13. MONTES Segundo: -""El agro salvadoreño" - UCA Editores, S.S., 1986.

14. PROCESO Ne 312, 18 de noviembre de 1987, UCA, San Salvador.

15. ROSIER Bernard: - "Structure agricole et développement économique" - Mouton, Paris, 1968.

16. SECONAN: - "Situación alimentaria nutricional"— San Salvador, marzo 1985.

17. TOUZARD Isabelle et Jean-Marc: - "Etude d'un système agraire:

San Dionisio, departamento de Matagalpa, Nicaragua"-Tesis, CNEARC, Montpellier, 1986.

18. CROZIER Michel, Friedberg Erchard: -"L'acteur et le systeme"- ed. Points-politique, Paris, 1974.

\section{ANEXO 1}

\section{RECOMENDACIONES PRELIMINARES}

\section{Procedimiento general}

En realidad, esta guía es un hilo director que permite animar o reanudar una discusión que debe ser abierta. Sirve también para recolectar los elementos necesarios para los cálculos económicos y así poner en evidencia los resultados de la explotación = la guía permite no olvidar ninguno de estos elementos significativos.

Es importante llevar a acabo una encuesta según distintos niveles de acercamiento de la explotación estudiada: 
-Bien apuntar las palabras propias del campesino y sus respuestas a las preguntas.

- Ser muy observador de la explotación y de los elementos indicadores del nivel de vida, de las diferentes relaciones entre los miembros de la familia (toma de decisión, división sexual del trabajo...), y relaciones entre la familia y el distrito de riego,...).

- Saber relativizar las respuestas, reubicándolas con el hecho que nosotros llevamos deformación en ellas = los agricultores nos asimilan a controladores, o miembros de organizaciones humanitarias.

- Hacer preguntas "cruzadas" para verificar una respuesta que parece en ruptura con el resto de la conversación.

\section{Etapas de la encuesta}

a) En la primera visita, orientar la discusión sobre los recursos de la explotación y la familia. Tratar de acercar todos esos elementos de la explotación de manera histórica, para cada vez saber el cuando de un acontecimiento, el porque, el como (con que dinero...).

Eventualmente, hacer un primer acercamiento de los sistemas de cultivo y/o ganaderia.

NOTA: Es muy importante saber porque el agricultor vino a Zapotitán, con que dinero compró la tierra, y cual fue la evolución de sus cultivos desde su llegada aquí.

b) En las segundas, y tercera visitas, se trata más que todo de visitar todas las parcelas, lo que permite afinar el estudio de los sistemas de producción. Es primordial entender el porque de las opciones técnicas del agricultor (porque este cultivo y no otro?, porque asociación de cultivos y no monocultivo? porque el uso del tractor y no del arado? porque el uso del riego o no?...).

También es muy importante establecer un calendario de trabajo para cada parcela y conocer la sucesión de los cultivos dentro del año, y de un ano al otro, en cada una.

- Tratar de elaborar con el productor un pequefío balance de los recursos/gastos para el año 1986 y un calendario aproximativo de trabajo (revelar eventualmente los picos de trabajo).

-Discutir con el productor de sus proyectos a corto y largo plazo.

-Pedirle jerarquizar sus problemas.

-Es importante de chequear los datos recolectados después de cada visita para pedir to que hace falta en la próxima visita.

-Pedir el nombre del productor al fin de la encuesta. 
- No olvidar presentarse al inicio de la encuesta, y explicar porque hacen este trabajo.

\section{GUIA DE ENCUESTA}

1. Datos generales sobre la fuerza de trabajo y los recursos de la explotación

\subsection{Fuerza de trabajo y consumldores}

\subsection{1: La familla:}

1) Composición: Número. Para cada una: sexo, edad, nivel escolar, donde vive, casados, viudos, servicio militar...

Tratar de sacar el número de consumidores (distinguir mayores-menores de 10 años).

2) Personas trabajando en la explotación: número para cada una: tareas agrícolas, hogarefias u otras (con calendario).

Tratar de sacar la división del trabajo (sexo, edad, parentesco).

3) Personas trabajando fuera de la explotación:

Número. Para cada una: tipo de trabajo, fecha (con calendario), lugar, duración, tipo de contrato (salario, mano vuelta...) condiciones laborales.

Estimar los ingresos de la familia fuera de la explotación.

\subsection{2: Mano de obra externa:}

1) Número de empleados permanentes. ¿Quiénes son?, cuando trabajan, para qué, tipo de contrato, salario (no olvidar los pagos en comida y otros).

2) Existencia de mano vuelta: cuándo, con quién, para qué.

3) Número de empleados temporales: quiénes son, cuándo trabajan, para qué, tipo de contrato, salario (no olvidar los pagos en comida y otros).

Estimar la cantidad de trabajo que provee la mano de obra externa.

Tratar de establecer un calendario de actividades de la mano de obra externa. 


\subsection{Las tierras}

Preguntar si el agricultor tiene otras tierras fuera del Distrito de Zapotitán, o casas, empresas, maquinaria.

Determinar a lo largo de la encuesta si hay flujos de capital entre Zapotitán y las demás actividades (o otros flujos, semilla, abono natural, etc.).

Determinar la especificidad de las actividades en Zapotitán.

1.2.1: Superficle total y por cultivo 0 asociación de cultivos en Zapotitán.

Hacer un croquis de la explotación.

1.2.2: Para cada parcela: (ir en cada una): tamaño, ubicación con respecto a la casa, a los caminos, a los canales de riego.

1) Suelos: Evaluar profundidad

determinar presencia de piedras

evaluar contextura.

2) Agua: - Presencia de fuentes, pozos, ríos (fuera de la infraestructura del Distrito de riego).

- Presencia de agua (charcos, tierras, empantanadas, lodo)

Infraestructura - Canales de irigación: estado, funcionamiento, fedel Distrito de Riego. chas del riego. Si ya no hay riego, desde cuando. Sistema de drenaje: estado, funcionamiento.

3) Tipo de tenencla de la tierra: propia, arrendada, colonato, decreto 207 , etc...

- Costo acceso

- fecha compra o de inicio de la actividad.

- Herencia?

4) Cultivos: Tipo, edad, calidad, densidad en $\mathrm{mz}$, o ha. (precisar)

5) Presencia o no de cercas - Tipo de cercas

\subsection{Otro capital}

\subsection{1: Ganado}

1) Tipo de animales, edad, raza, tenencia. 
2) Adquisición: fecha, precio, vendedor.

3) Propósito.

4) Inventario ventas del año y precio.

5) Historia del ganado: cambios importantes: de raza, de propósito, de tenencia. Origen de estos cambios.

\subsection{2: Infraestructura de la explotación}

1) Tipo de capital, número, edad, estado, modo de adquisición.

2) Utilización (alquilado a veces?) ¿Hace falta otra infraestructura?

3) Fecha adquisición, origen dinero, como se consiguió el material.

\subsection{3: Herramienta y vehículos}

1) Tipo, número, edad, estado, modo de adquisicón.

2) tilización. Hace falta otra infraestructura?

3) Fecha de adquisición, origen dinero, como se consiguió el material.

\subsection{Acceso al agua.}

1) Costo legal y real.

2) Trámites necesarios.

3) Relaciones con la oficina y el canalero.

4) Organización del riego. Participación al mantenimiento.

1.5. Acceso al capital financiero.

1) Capital bancario o cooperativas de crédito:

- Tipo de préstamos (plazo-interés).

- Condiciones/garantías.

- Estimación deuda actual.

- Eventuales problemas de reembolso.

2) Capital de los prestamistas:

- Tipo de préstamo (plazo/interés).

- Condiciones de préstamo-garantías.

- Estimación deuda actual.

- Problemas de reembolso.

1.6. Acceso a la información

- Asesoría técnica/extensión agrícola: acerca de que cultivos. Consejos.

- Diarios/radio.

- Asistencia a las reuniones del distrito. 
2. Historia de la familia y de la explotación

- Orígen de los padres y de los abuelos: (lugar-actividad)

- Historia del agricultor: - Primer trabajo independiente.

- Sucesión de trabajos (lugar-actividad)

- Porqué esos movimeintos.

- Llegada en Zapotitán (actividades porqué?)

- Fecha del casamiento: papel eventual de la esposa en la adquisición de tierra.

- Tratar de vincular los nacimientos con una fase de acumulación de capital).

- Modalidades de compra-venta de capital (ganado, máquina, etc...) vinculándolas con la familia.

- Fecha del primer trabajo de los hijos. Que hacen ahora.

- Evolución de la deuda familiar. A quién prestó el dinero?

\begin{tabular}{|c|c|c|c|c|c|c|c|c|}
\hline \multirow{2}{*}{$\begin{array}{l}\text { Trabajo reall- } \\
\text { zado (ciembra, } \\
\text { limpleza, abo- } \\
\text { nado, cosecha, } \\
\text { almacena- } \\
\text { miento...) }\end{array}$} & \multirow{2}{*}{$\begin{array}{l}\text { Focha } \\
\text { del } \\
\text { traba- } \\
10\end{array}$} & \multirow{2}{*}{$\begin{array}{l}\text { Herra- } \\
\text { mienta } \\
\text { utilizada }\end{array}$} & \multicolumn{4}{|c|}{$\begin{array}{l}\text { Inaumo (abono, comillia, } \\
\text { plagulcida, agua, tutor, otc.) }\end{array}$} & \multirow{2}{*}{$\begin{array}{l}\text { Cantidad } \\
\text { do } \\
\text { trabajo } \\
\text { diace- } \\
\text { nombre }\end{array}$} & \multirow{2}{*}{$\begin{array}{l}\text { Notas } \\
\text { (condi- } \\
\text { conos } \\
\text { de } \\
\text { trabajo, } \\
\text { otc. }\end{array}$} \\
\hline & & & $\begin{array}{l}\text { lugar } \\
\text { do } \\
\text { compra }\end{array}$ & Precio & dosis & $\begin{array}{l}\text { credl } \\
\text { to }\end{array}$ & & \\
\hline & & & & & & & & \\
\hline & & & & & & & & \\
\hline & & & & & & & & \\
\hline & & & & & & & & \\
\hline & & & & & & & & \\
\hline & & & & & & & & \\
\hline & & & & & & & & \\
\hline & & & & & & & & \\
\hline & & & & & & & & \\
\hline & & & & & & & & \\
\hline & & & & & & & & \\
\hline
\end{tabular}


- Accidentes especiales. Influencia de la guerra.

\section{Sistema de producción}

\subsection{Los cultivos y plantaciones}

Para cada cultivo o asociación de cultivos:

- Superficie sembrada

- El cultivo y su variedad

\section{Es imperativo conocer:}

- La estancia de animales en la parcela.

- Detectar los picos laborales y la eventual competencia entre los cultivos.

- Las rotaciones de cultivo: preguntar cual va a ser el próximo cultivo y cual fue el anterior.

- Los problemas agronómicos. Enfermedades (desde cuando?) malezas problemas de agua.

- Los problemas de insuficiencia de mano de obra.

- Cual es la fertilidad aproximada (pasan animales después de la cosecha?).

- Los rendimientos. Estimación ¿Estabilidad?

- La comercialización.

- Cuál es la repartición autoconsumo-venta? (dentro del auto-consumo, especificar: Alimentación humana, animal, semillas...).

- Precio de venta.

- Quién compra.

- Modalidades de venta: contrato, al peso, según la calidad, etc.

- Calendario de ventas (ingrsos moentarios). Almacenamiento.

\subsection{Los barbechos (eventuales)}

- Desde cuando esta parcela es un barbecho.

- Ultimos cultivos.

- Cuando va a ser la parcela de nuevo cultivada.

- Porque esta parcela es un barbecho.

- Utilización del barbecho (leña, pasto).

\subsection{La ganadería}

\subsection{1: Los pastos (estudiar cada parcela)}

- Desde cuando ésta parcela es un pasto.

- ¿Que hacía antes? 


\begin{tabular}{|c|c|c|c|c|}
\hline $\begin{array}{c}\text { TRABAJOS } \\
\text { REALIZADOS }\end{array}$ & $\begin{array}{c}\text { FECHAO } \\
\text { FRECUENCIA }\end{array}$ & $\begin{array}{c}\text { INSUMOS(c/cultivo } \\
\text { (idem cultivos) }\end{array}$ & CANTIDAD & $\begin{array}{c}\text { QUIEN } \\
\text { TRABAJA }\end{array}$ \\
\hline $\begin{array}{c}\text { - Instalación } \\
\text { - Mantenimiento }\end{array}$ & & & & \\
- Cortes \\
(Totales o \\
parciales) \\
Ensilage
\end{tabular}

- Corte Total: El ganado nunca está en la parcela

Parcial: El ganado a veces pasta, pero hay cosechas.

Estimas el rendimiento del paso si es posible.

- Cuales son las variedades presentes: variedades seleccionadas y espontáneas.

- Estimar el estado del pasto (mantenimiento, sobre utilización).

- Problemas de agua. Evaluación de las reservas de agua del pasto (río, fuente...)

- ¿riego?

- ¿abrevaderos? Distancia, cantidad.

- Estancia de animales.

- Fecha de inicio y de fin del paso. Frecuencia.

- Cantidad y tipo de animales para cada período.

- Eventualmente: quienes son los dueños de los animales.

- Carga animal/Ha: Detectar la rotación del ganado en los pastos.

- Otros Pastos (estabulación)

- Cuando se lleva el forraje a los animales.

- Frecuencia.

- Cantidad aproximada/tipo de animal.

- Quien se encarga de picary de dar de comer al ganado.

\subsection{2: El ganado}

1) Los distintos tipos de producción (leche, carne, animales (edad) 
2) Destino de la producción.

- Establecer para el año 87 las comprasy ventas de animales: fechas, cantidades, precios.

- Con que dinero las compras se hicieron (crédito?).

- Como se utiliza el dinero de las ventas?

- Quién compra los productos de la ganadería. Regularidad de las ventas.

- Transporte de animales, de la leche, quién lo hace y a que precio.

- Papel del autoconsumo (leche, carne). Cantidades.

\section{3) Reproducción del Hato}

- Titpo de monta (en estación, inseminación artificial, monta libre).

- Cruces (controlados o no).

- Si es posible: calendario de las pariciones.

- Intervalo de las pariciones.

\section{4) Estado sanitario del hato.}

- Inventario de las muertes de animales ocurridos en 1986. Causa.

- Enfermedades. Como se curan.

- Vacunaciones. Frecuencia.

- Tratamientos preventivos y curativos de los parásitos internos o externos.

- Cada vez preguntar el precio de los medicamentos o vacunas, (lugar de compra).

- Existencia de asesoria técnica, visitas del veterinario.

\section{5) Alimentación}

- Forraje, pastos: estudiarles en el punto 3.3.1.

- Compra de alimentos complementarios: concentrados, sal, vitaminas, miel,...

Cantidad

Precio

Origen

- A cuales animales se dan estos productos.

6) Estabulación (y eventualmente ordefiadora)

- ¿Qué se hace con estiércol?

- La pajaza de donde viene. Precio. 


\subsection{Ganadería de corral}

- Mismas preguntas que en el 3.3.2

- Estudiar las formas de compra de insumos y de productos diversos. Contrato?

4. Proyectos y reivindicaciones

- Proyectos: Tipo, recurso, plazo (corto, mediano, largo).

- Relaciones con el Estado.

- Relaciones y reivindicaciones con respecto al Distrito de Riego.

- Pertenece a una organización campesina. Responsabilidades

- Cuales son para él sus principales limitaciones. 